\title{
Ring-Current Assessment of the Annulene-Within-an-Annulene Model for some Large Coupled Super-Ring Conjugated-Systems ${ }^{\dagger}$
}

\author{
Timothy K. Dickens ${ }^{\mathrm{a}}$ and Roger B. Mallion ${ }^{\mathrm{b}, *}$ \\ ${ }^{a}$ University Chemical Laboratory, University of Cambridge, Lensfield Road, Cambridge \\ CB2 1EW, England, United Kingdom \\ ${ }^{\mathrm{b}}$ School of Physical Sciences, University of Kent, Canterbury \\ CT2 7NH, England, United Kingdom
}

RECEIVED NOVEMBER 20, 2013; REVISED MAY 18, 2014; ACCEPTED JULY 07, 2014

\begin{abstract}
The Annulene-Within-an-Annulene (AWA) model for conjugated super-ring systems, proposed by Barth and Lawton nearly fifty years ago, is further tested on six newly considered 'coupled' structures by means of $\pi$-electron ring-currents and bond-currents calculated via the Hückel-London-PopleMcWeeny (HLPM) formalism. Super-ring systems are said to be decoupled when the bonds connecting the central ring to the outer perimeter never appear as anything other than single bonds in any Kekulé structure that can be devised for the system as a whole. The preliminary conclusions of other recent investigations - by the same HLPM method and by ipso-centric ab-initio approaches - are verified. Phenylene-[5]-circulene, a (coupled) alternant isomer of the much-studied (decoupled) non-alternant system [10,5]coronene, is also considered. It is advised that the AWA model should in future either be used with considerable caution in very specific circumstances, or it should be abandoned altogether.
\end{abstract}

Keywords: HLPM ring-currents \& bond-currents, graph theory, conjugated hydrocarbons, coupled \& decoupled super-ring systems, annulene-within-an-annulene rule

\section{INTRODUCTION}

A recent review in this journal ${ }^{1}$ has provided step-bystep insight into the practical details of calculating what the present authors call 'topological ${ }^{2-4} \pi$-electron ringcurrents and bond-currents in conjugated hydrocarbons, by means of the Hückel ${ }^{5}-$ London $^{6}-$ Pople $^{7}-\mathrm{McWeeny}^{8}$ (HLPM) formalism. As was argued there (and elsewhere ${ }^{9,10}$ ), such ring currents and bond currents - unlike those evaluated from more-sophisticated approaches, including ab-initio ones - have the distinct conceptual advantage that they depend solely on (i) how the carbon-atoms in the network that constitutes a given conjugated system are connected to each other by $\sigma$-bonds and on (ii) the areas of the hydrocarbon's constituent rings. No knowledge or assumptions of any other parameters are required for an application of the HLPM $^{1-8}$ method. ${ }^{11,12}$

The present authors have applied this technique to a wide variety of extant and hypothetical conjugatedsystems, ${ }^{1,13-17}$ primarily in order to test the so-called 'Annulene-Within-an-Annulene (AWA) Rule'1,13-29 for what have been described as 'super-ring' structures. $1,30-33$
A general super-ring conjugated hydrocarbon is illustrated in Figure 1 (which is taken from Ref. 1.)

Such a system is said to obey the AWA rule, insofar as its magnetic properties are concerned, if its inner

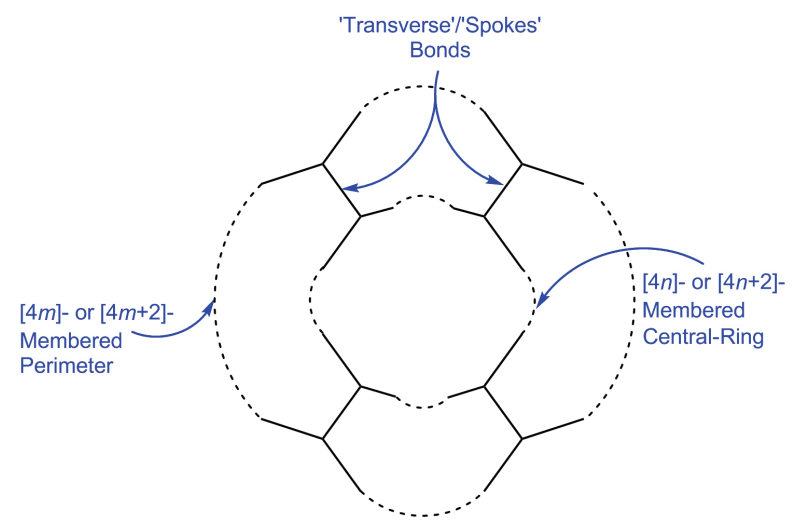

Figure 1. Schematic depiction of a general 'super-ring' structure, ${ }^{1,30-33}$ showing the central ring (usually $[4 n]$ - or [4n+2]-membered), the outer perimeter (usually $[4 m]$ - or $[4 m+2]-m e m-$ bered), and the "transverse ${ }^{30} /$ spokes $^{34}$ bonds that connect the inner ring and the outer perimeter.

\footnotetext{
$\dagger$ Respectfully dedicated to Professor Roy McWeeny, ring-current pioneer, on the occasion of his $90^{\text {th }}$ birthday (May 19 ${ }^{\text {th }}$, 2014).

* Author to whom correspondence should be addressed. (E-mail: R.B.Mallion@kent.ac.uk)
} 
ring and its outer perimeter behave as if they were independent annulenes by exhibiting a current flow in the diamagnetic (anti-clockwise) direction if these cycles are $[4 p+2]$-membered and in the paramagnetic (clockwise) sense if they are $[4 p]-$ membered ( $p$ being an integer), when the structure is in the presence of an external magnetic-field, considered to be directed at right angles to the (assumed) molecular plane.

In Ref. 1, the seven super-ring structures discussed by Aihara in Ref. 32 were all shown not to respect the AWA rule when tested by the HLPM approach. They failed for various different reasons and, indeed, the general conclusion was that, at least when tested against HLPM $^{5-8}$ ring-currents and bond-currents, most superring structures ${ }^{1,30-33}$ do not ${ }^{1,13,14}$ respect the AWA 'rule'; certain carefully 'designed ${ }^{23-29}$ and occasionally somewhat artificial structures ${ }^{15,16}$ do, ${ }^{1,15,16,23}$ however. Furthermore, similar overall conclusions have largely been drawn when the AWA rule has been tested against the predictions of much more sophisticated 'ipso-centric' ab-initio calculations ${ }^{20-23,35}$ - please see Ref. 36 for a detailed history of this approach - and of those $\mathrm{e}^{34,37,38}$ based on the 'pseudo- $\pi$ ' approximation, ${ }^{37}$ as well as on predictions obtained via more-rudimentary methods (not considered in this paper), which make appeal to the idea $^{12,39-46}$ of what Randic ${ }^{47}$ called 'conjugated circuits' (defined in Ref. 47). These were independently applied to ring-currents by Gomes ${ }^{48}$ and subsequently referred to by the latter author as 'circuits of conjugation' (defined in Refs. 48-50).

It should, however, be pointed out in passing that, in the case of 7-coronene (structure (II) of Ref. 15), conclusions based on the topological ${ }^{1-3}$ HLPM approach are 'at odds ${ }^{29}$ with those drawn from an application of more-sophisticated ab-initio formalisms. ${ }^{29}$ Furthermore, such calculations tend frequently to be based (more realistically) on buckle-shaped molecules (e.g., Ref. 29), rather the planar structures that are intrinsic ${ }^{1}$ to the 'topological' HLPM approach; ${ }^{1-4}$ hence, for this reason, let alone any quantum-mechanical ones, it is perhaps not surprising that divergent results - e.g. those indicated in Refs. 29 and 15 - can sometimes arise. ${ }^{15,29}$ (Please see the Appendix for a discussion on the question of (geometrical) non-planarity.) Most ${ }^{1,15,16,23}$ (though not all $^{1,13,34}$ ) of those conjugated systems so far encountered that do respect the AWA rule are super-ring structures in which all the peripheral rings (see Figure 1) are symmetrically equivalent — such as the $p$-coronenes defined in Ref. 15 and one of the $[r, s]$-coronenes discussed in Ref. 16, and structure 8 of Ref. 1. That said, we emphasise that we strongly concur ( $c f$. Ref. 1) with the view of Monaco and Zanasi ${ }^{26}$ that the symmetry enforcement of zero bond-current in the 'spokes' bonds $^{34}$ (see Figure 1) is insufficient to infer that the inner and outer cycles of such structures behave independently, as the AWA model requires.

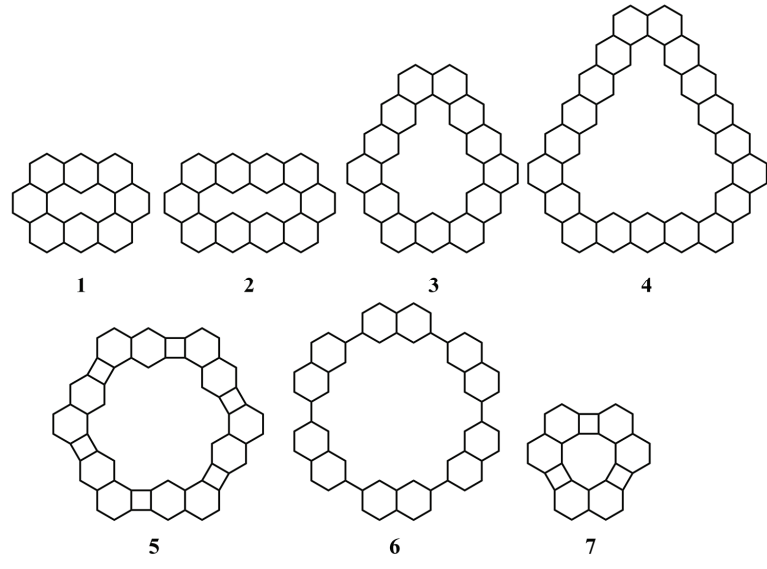

Figure 2. The carbon-atom skeletons (molecular graphs) of the seven 'super-ring' structures initially considered in this study.

In the present work, we apply the HLPM meth$\mathrm{od}^{1,5-8}$ to calculate bond currents in the central rings and outer perimeters in seven structures $(\mathbf{1}-\mathbf{7})$ not previously considered by us, which are depicted in Figure 2; two of them (1 and 2) are potentially [4n+2]-annuleneswithin-[4m+2]-annulenes - for nomenclature please see Ref. 1 and Figure 1 - three $(\mathbf{3}, \mathbf{4}$ and $\mathbf{5})$ are candidates for being a $[4 n]$-annulene-within-a- $[4 m]$-annulene, one (6) is a peculiar species analogous to structure 4 of Ref. 1, and one (7) has odd-membered inner and outer rings and hence merits further investigation and discussion. At the very end of the paper, we shall consider the ring-current and bond-current maps of a coupled alternant isomer of the much-discussed ${ }^{15,16,23,25,27,30}$ (but decoupled) [10,5]-coronene.

\section{CALCULATIONS}

HLPM ring-current intensities were calculated according to the method described in complete detail in Ref. 1, by use of equation (14) of that reference. The prescription $^{1-4}$ for evaluating strictly 'topological' ${ }^{1-4}$ ringcurrents is that the areas of the constituent rings of various sizes in the conjugated structures under study are estimated (by equation (16) in Ref. 1) on the assumption that they are regular polygons of the same side-length. However, five of the benzenoid hydrocarbons studied here (structures 1-4 and $\mathbf{6}$ of Figure 2) possess central rings which, geometrically speaking, are effectively a 'hole' in an otherwise perfectly tessellated hexagonal lattice. Accordingly, as was the case in Ref. 1, the areas of the central rings in these five structures are estimated as being the total area of the 'missing' hexagons in each structure. These central rings are thus taken to have an area of two benzene-hexagon units in the case of $\mathbf{1}$, three benzene-hexagon units in the case of 2 , eight benzene-hexagon units in the case of $\mathbf{3}$ and nineteen benzene-hexagon units for $\mathbf{4}$ and $\mathbf{6}$. 
Table 1. Qualitative summary of the HLPM calculations on structures 1-7

\begin{tabular}{|c|c|c|c|c|c|}
\hline $\begin{array}{c}\text { Structure } \\
\text { (Labelled and } \\
\text { Displayed in } \\
\text { Figure 2) }\end{array}$ & $\begin{array}{l}\text { Number of Car- } \\
\text { bon Atoms in the } \\
\text { Outer Periphery }\end{array}$ & $\begin{array}{l}\text { Number of Car- } \\
\text { bon Atoms in the } \\
\text { Central Ring }\end{array}$ & $\begin{array}{c}\text { Nature of the } \\
\text { Individual Bond- } \\
\text { Currents for Bonds on } \\
\text { the Outer Periphery }\end{array}$ & $\begin{array}{c}\text { Nature of the } \\
\text { Individual Bond- } \\
\text { Currents for Bonds in } \\
\text { the Central Ring }\end{array}$ & $\begin{array}{c}\text { Is This } \\
\text { Compliant } \\
\text { with the } \\
\text { AWA Rule? }\end{array}$ \\
\hline $\mathbf{1}^{\text {(a) }}$ & $\begin{array}{c}22 \\
(4 m+2)\end{array}$ & $\begin{array}{c}10 \\
(4 n+2)\end{array}$ & Diamagnetic & Paramagnetic & NO \\
\hline $2^{(a)}$ & $\begin{array}{c}26 \\
(4 m+2)\end{array}$ & $\begin{array}{c}14 \\
(4 n+2)\end{array}$ & Diamagnetic & Paramagnetic & NO \\
\hline $\mathbf{3}^{(\mathrm{a})}$ & $\begin{array}{c}32 \\
(4 m)\end{array}$ & $\begin{array}{c}20 \\
(4 n)\end{array}$ & Diamagnetic & Paramagnetic & NO \\
\hline $4^{(a)}$ & $\begin{array}{c}44 \\
(4 m)\end{array}$ & $\begin{array}{c}32 \\
(4 n)\end{array}$ & Diamagnetic & Paramagnetic & NO \\
\hline $5^{(\mathrm{b})}$ & $\begin{array}{c}36 \\
(4 m)\end{array}$ & $\begin{array}{c}24 \\
(4 n)\end{array}$ & Mixed & Mixed & NO \\
\hline $6^{(\mathrm{b})}$ & $\begin{array}{c}42 \\
(4 m+2)\end{array}$ & $\begin{array}{c}30 \\
(4 n+2)\end{array}$ & Diamagnetic and Zero & $\begin{array}{c}\text { Diamagnetic and } \\
\text { Zero }\end{array}$ & $\begin{array}{l}\text { Please see } \\
\text { Discussion }\end{array}$ \\
\hline $7^{(\mathrm{b})}$ & $\begin{array}{c}21 \\
(4 m+1)\end{array}$ & $\begin{array}{c}9 \\
(4 n+1)\end{array}$ & Paramagnetic & Diamagnetic & $\begin{array}{l}\text { Please see } \\
\text { Discussion }\end{array}$ \\
\hline
\end{tabular}

${ }^{(a)}$ A long name has been suggested for structure $\mathbf{1}$ in Ref. 51, where it is referred to as cyclo[d.e.e.e.d.e.e.e.]-octakisbenzene. Alternative names of this type could probably be similarly devised for structures $\mathbf{2}, \mathbf{3}$ and $\mathbf{4}$, if desired.

(b) Names for structures $\mathbf{5}$ and $\mathbf{7}$ have been suggested by Balaban and Vollhardt in Ref. 52 and Nakanishi et al. named structure $\mathbf{6}$ in Ref. 53.

All the calculations reported here are based on branched spanning-trees and (with one exception, mentioned later, when considering one of several sets of calculations on structure 6) were carried out in Double Precision. (Please see Ref. 1 for a detailed discussion of the relevance of these more arcane aspects of the calculations by the HLPM approach. ${ }^{1-8}$ )

Some of the structures 1-7 might possibly be nonplanar - either as a result of proton-proton steric interactions and/or because of angle strain arising from too large a deviation from the idealised $s p_{2}$-hybridised $120^{\circ}$ $\mathrm{C}-\mathrm{C}-\mathrm{C}$ angles. This question is pursued, using struc- tures 1 and 2 as exemplars, in the Appendix. As previously emphasised $^{1,2}$ the HLPM ring-currents calculated for any conjugated systems dealt with here that might be non-planar are, in effect, those for a hypothetical structure having the same carbon-carbon connectivity as the actual molecule under consideration, but envisaged as if it were geometrically planar.

HLPM ring-current and bond-current intensities calculated in this way for structures 1-7 are displayed in Figures 3, 5-7, and 9; they are expressed, as is conventional, ${ }^{1}$ as a (dimensionless) ratio to the ring-current intensity calculated, by the same method, for the unique
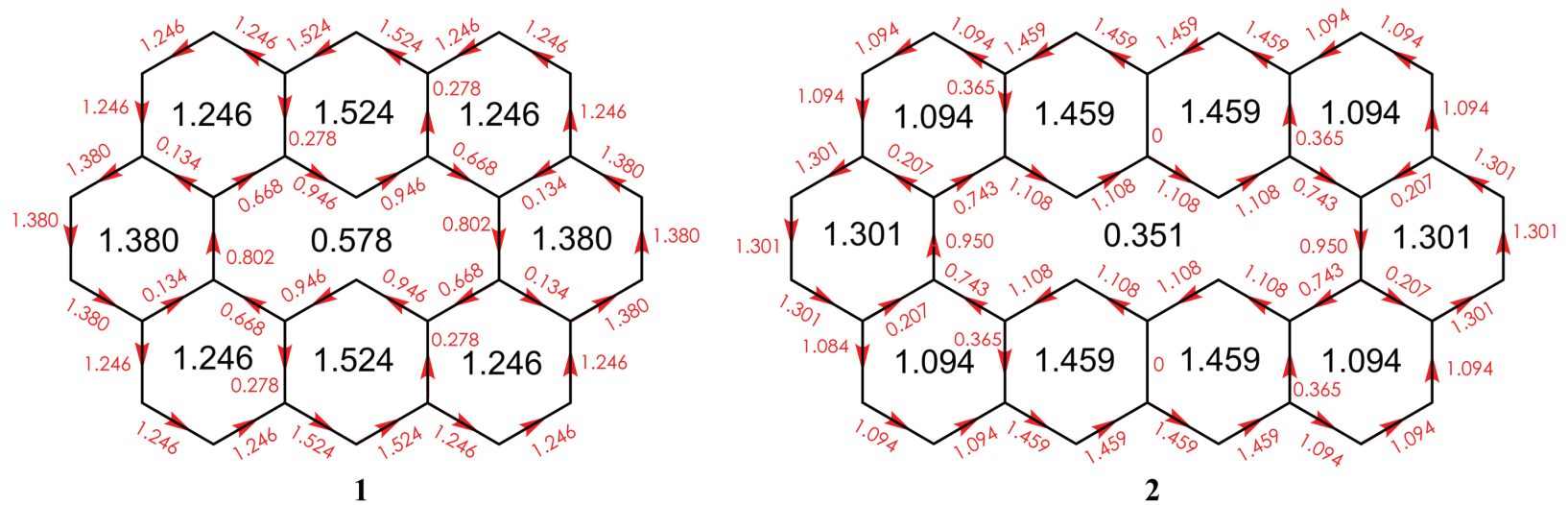

Figure 3. Ring-current and bond-current maps for structures 1 and 2. Ring-currents (in black) and the associated bond-currents (in red, in the on-line edition of the journal) were calculated by the $\mathrm{HLPM}^{5-8}$ approach, as described in the text. The ring-currents and the bond-currents are dimensionless quantities; accordingly, all are appropriately depicted here as pure numbers, without units. Positive (diamagnetic) ring-currents are considered to circulate anti-clockwise around their respective rings whilst negative (paramagnetic) ring-currents flow in the clockwise sense around those rings. The numerous bond-currents flow in the direction indicated by the arrow pointing along each bond. 
ring in benzene. In all the current maps presented in this paper, ring-current intensities are written (in black) in the centre of each ring; when positive (that is, diamagnetic), they are considered to be circulating in the anticlockwise sense around the ring in question: when negative (paramagnetic), they are regarded as flowing in the clockwise direction around that ring. The corresponding bond-currents are written (in red, in the on-line edition of the journal) along the respective bonds, and they flow in the direction indicated by the arrow on each bond. Bond currents have been deduced from the ring currents - calculated as described in Ref. 1 - by repeated application of the microscopic analogy of Kirchhoff's Law of Conservation of Currents at Junctions in macroscopic electrical networks. ${ }^{1,12,50}$ The qualitative nature of the current flows in structures 1-7 is summarised in Table 1.

\section{DISCUSSION OF RESULTS}

Structures 1 and 2 (Figure 3). These are both conjugated super-ring systems having [4n+2]-rings within [4m+2]rings. In the case of $\mathbf{1}$ there is a [10]-ring within a [22]ring and 2 has a [14]-membered ring within a [26]membered ring. All the outer rings in $\mathbf{1}$ and $\mathbf{2}$ bear a diamagnetic ring-current and so the unshared bonds in these rings, which form the outer, [22]-membered (1) or [26]-membered (2) perimeter, all carry diamagnetic currents, as indicated in Figure 3. This is as would be expected if $\mathbf{1}$ and $\mathbf{2}$ obeyed the AWA rule. However, although the central rings of $\mathbf{1}$ and $\mathbf{2}$ also both have associated with them diamagnetic ring-currents, such currents in these central rings are, in both cases, much smaller in magnitude than those in the peripheral rings in $\mathbf{1}$ and $\mathbf{2}$ with which the central rings share at least one bond. The result of the electronic competition in the bonds that the central rings of $\mathbf{1}$ and $\mathbf{2}$ share with the outer rings of these structures means that bonds in the central rings of both systems bear net currents which, from the point of view of the central rings, flow in the paramagnetic (clockwise) direction around those rings. This is indicated in the bond-current maps for structures $\mathbf{1}$ and $\mathbf{2}$ depicted in Figure 3. Such a paramagnetic circulation in the central rings is contrary to the requirements for these structures to behave as an annulene-within-anannulene. 1 and $\mathbf{2}$ therefore do not comply with the AWA rule.

Before moving to the other super-ring ${ }^{1,30-33}$ structures (3-7) depicted in Figure 2, we just observe in passing that the structures $\mathbf{1}$ and $\mathbf{2}$ (which, so far as we know, are hypothetical) whose current maps have just been presented in Figure 3, are formally related - by the suppression of two (structure 1) or four (structure 2) protons and the concomitant addition of one (structure 1) or two (structure 2) carbon-carbon bonds - to the well-known and characterised ${ }^{54,55}$ planar condensed, benzenoid hydrocarbons ovalene ${ }^{54}(8)$ and circumanthracene $^{55}$ (9), respectively; the HLPM topological current-maps of these structures - which, it should be noted, are not actually 'super-ring' ones ${ }^{1,30-33}$ — are presented in Figure 4. The topological ring-current intensities in ovalene (8) - derived from SinglePrecision ${ }^{1}$ calculations based on a continuous ('unbranched') spanning-tree ${ }^{1}$ — have already been reported in Ref. 2. We have, however, recomputed them basing the calculation on a branched spanning-tree and carrying out the required manipulations in Double Precision. Please see an extensive discussion in Ref. 1 for the relevance of such matters in this context. The recalculated values are all within three digits in the third decimal-place of those reported in Ref. 2.

The peripheral rings in $\mathbf{8}$ and $\mathbf{9}$, like their counterparts in $\mathbf{1}$ and $\mathbf{2}$, do (as expected) bear avowedly diamagnetic ring-currents of the size routinely encountered in the condensed, benzenoid hydrocarbons. ${ }^{2}$ The 'extra' central-rings in both 8 and $\mathbf{9}$ that are created by the formal proton-deletions and carbon-carbon bondadditions in $\mathbf{1}$ and $\mathbf{2}$, respectively, just described, also bear ring currents of an intensity ( $c a$. 1.3-1.7, in these cases) typical of those routinely associated with the corresponding rings in other condensed, benzenoid hydrocarbons. ${ }^{2}$ This is in stark contrast to $\mathbf{1}$ and $\mathbf{2}$, where the much larger central-rings $(10$-membered in structure 1, and 14-membered in structure 2) bear much lower ring-currents - 0.578 in the case of 1 and only 0.351 in the case of 2 . Despite their larger ring-areas, therefore, these central rings in $\mathbf{1}$ and $\mathbf{2}$ would make a much smaller contribution to the overall 'London' diamagnetic susceptibility of these structures than do the central rings in their respective counterparts, $\mathbf{8}$ and $\mathbf{9}$. On the basis of the ring currents reported in Figures 3 and 4, and the ring areas (and the planarity) of 1, 2, 8 and 9 being assumed here, the ratios

$$
\frac{\chi_{\perp}^{\pi \text {-London }}(\text { structure })}{\chi_{\perp}^{\pi \text {-London }} \text { (benzene) }}
$$

calculated, from equation (17) of Ref. 1, for the two pairs of structures ( $\mathbf{1}$ and $\mathbf{8})$ and (2 and $\mathbf{9})$, are: 11.95 (for 1) as against 14.85 (for 8) and 13.87 (for 2) as compared with 19.44 (for 9).

Structures 3 and 4 (Figure 5). Structures 3 and $\mathbf{4}$ consist of a $[4 n]$-ring within a $[4 m]$-ring — in the case of $\mathbf{3}$, there is a [20]-membered ring within a [32]-membered ring whilst, in 4, a [44]-membered periphery encloses a [32]-membered inner-ring. The peripheral rings in both 3 and 4, which each contribute either two or three bonds to the outer perimeter, all bear diamagnetic currents, and so the direction of flow around the perimeter is, in each case, anti-clockwise. Already, therefore, even before the central rings of $\mathbf{3}$ and $\mathbf{4}$ are considered, it is seen that the AWA rule is violated. We note, nevertheless, that there 

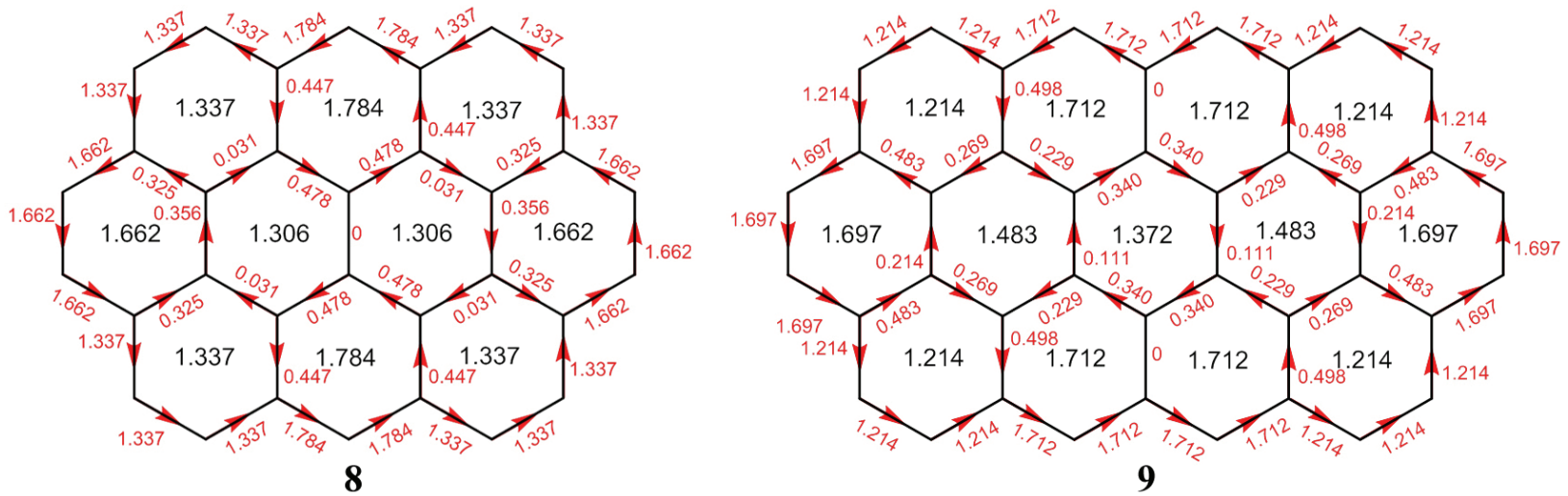

Figure 4. Ring-current and bond-current maps for structures 8 and 9 (the extant and well-characterised ovalene ${ }^{54}$ and circumanthracene, ${ }^{55}$ respectively). For conventions on the representation of ring currents (black) and bond currents — red, in the on-line edition of the journal - please see the caption to Figure 3.

is a small paramagnetic ring-current in the central rings of both 3 and $\mathbf{4}$, which would tend to drive current in the clockwise sense around these rings, and the currents in that direction in those shared bonds of the central rings are re-enforced by the diamagnetic ring-currents in the outer rings that form the structures' perimeter. The direction of current flow around the bonds of the central rings of $\mathbf{3}$ and $\mathbf{4}$ is thus clockwise (that is, paramagnetic), as is illustrated quantitatively in the bond-current maps for structures $\mathbf{3}$ and $\mathbf{4}$ shown in Figure 5. This is as it should be if $\mathbf{3}$ and $\mathbf{4}$ were to respect the AWA rule. However, as already noted, these structures fail the test of obeying the AWA model on account of the diamagnetic flows in their peripheries, just described.

Structure 5 (Figure 6). Like the benzenoid structures 14, structure 5 (named cyclic [6]naphthylene by Balaban and Vollhard ${ }^{52}$ ), consisting only of only even-membered rings, is an alternant hydrocarbon which thus fulfils the provisions of the Coulson-Rushbrooke Theorem. ${ }^{5,56} \mathrm{As}$ with structures 3 and 4, cyclic [6]naphthylene (5) consists of a $[4 n]$-ring within a $[4 m]$-ring; in this case, a [24]-membered inner-ring is situated within a [36]membered perimeter, as depicted in Figure 6. Cyclic [6] naphthylene (5) was recently discussed by Balaban and Vollhardt. ${ }^{52}$ The current pattern predicted here is dramatically different from that found in the benzenoid [4n]-within-[4m] alternant-hydrocarbons 3 and $\mathbf{4}$ (whose current maps are illustrated in Figure 5). The two types of symmetrically distinct six-membered rings on the periphery of 5 do all still bear diamagnetic ring-currents (though much smaller ones than those associated with the corresponding six-membered rings in 1-4); however, the six four-membered rings in $\mathbf{5}$, which each contribute one bond to the outer perimeter, support strongly paramagnetic ring-currents. The situation is therefore such that the 30 bonds on that part of the perimeter provided by the twelve six-membered peripheral-rings carry a current in the anti-clockwise (diamagnetic)
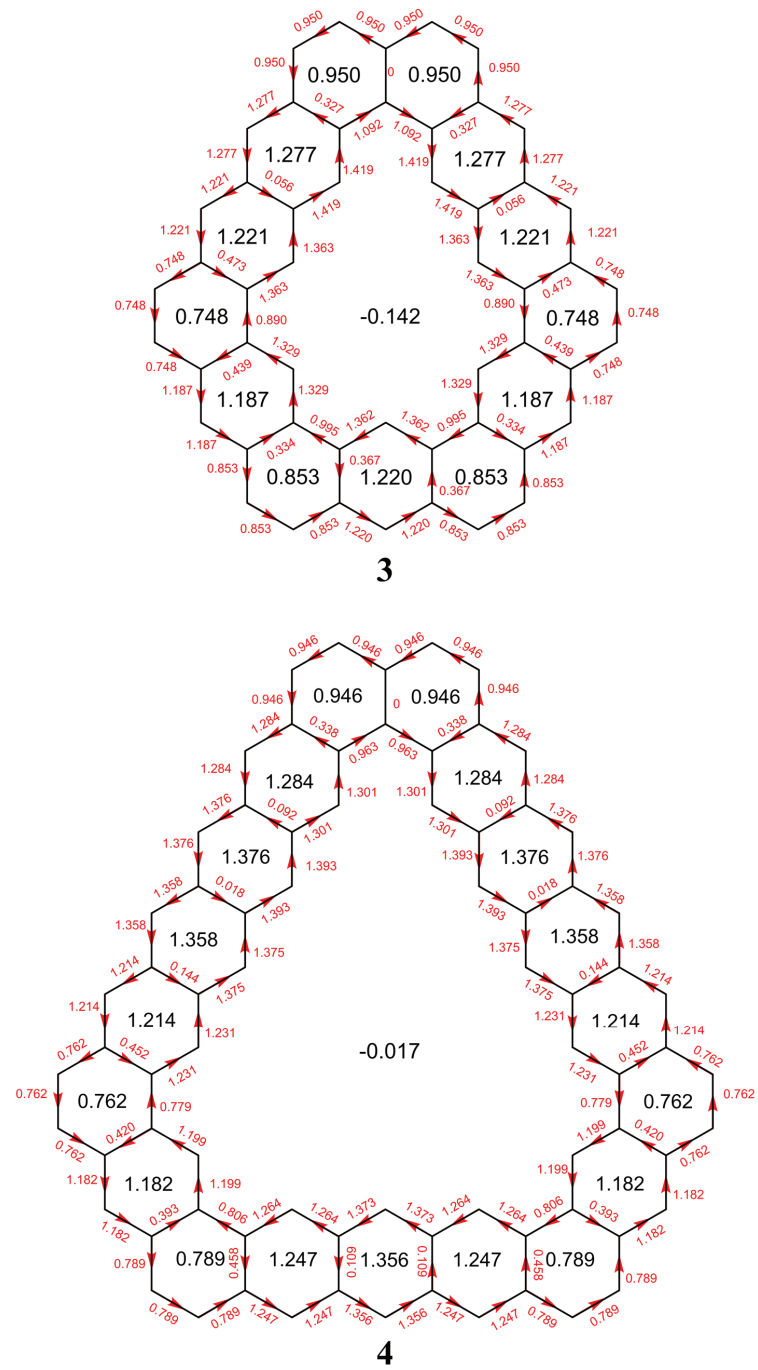

Figure 5. Ring-current and bond-current maps for structures 3 and 4. For conventions on the representation of ring currents (black) and bond currents - red, in the on-line edition of the journal - please see the caption to Figure 3. 


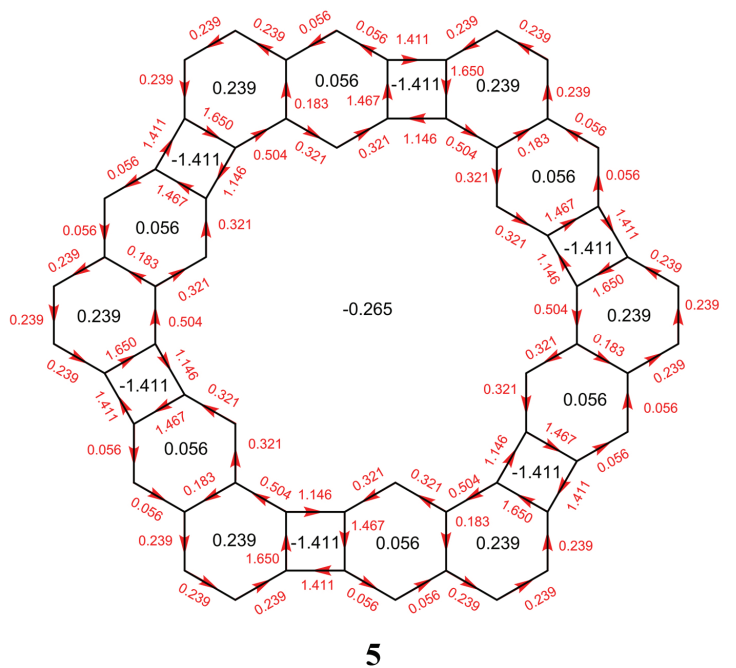

Figure 6. Ring-current and bond-current maps for structure 5 (named cyclic [6]naphthylene by Balaban and Vollhardt ${ }^{52}$ ). For conventions on the representation of ring currents (black) and bond currents - red, in the on-line edition of the journal — please see the caption to Figure 3.

sense, while the currents in the six bonds contributed to the perimeter by the six four-membered rings are in the clockwise (paramagnetic) direction around that perimeter; accordingly, in Table 1, the direction of current flow around the periphery of $\mathbf{5}$ is described as 'mixed'. This same conclusion was drawn in the case of antikekulene (structure 6 of Ref. 14, also known as cyclic [6]phenyl$\mathrm{ene}^{52}$ ), examined by the present authors, in the context of the AWA rule, in Refs. 14 and 1. (Antikekulene was also discussed by Balaban and Vollhardt in Ref. 52.) As a consequence, even before the central ring of $\mathbf{5}$ is considered, it is immediately clear that the AWA rule is violated by cyclic [6]naphthylene (5).

We nevertheless now do consider that central ring. It has associated with it a small paramagnetic current, on account of which, if it were the sole determinant of the matter, there would be a paramagnetic (clockwise) circulation around the bonds of the central ring. However, in those six bonds of the central ring that are shared with the four-membered surrounding rings (which each bear a strong paramagnetic ring-current), the small clockwise circulation (to the extent of 0.265 ) around the central ring is overwhelmed by a much larger current (of size 1.411) in the opposite direction, in the bonds of the central ring that are shared with the four-membered rings. This results in net currents in such central-ring bonds (of size $1.411-0.265=1.146$ ) that flow in the anti-clockwise (diamagnetic) direction from the point of view of the central ring. Exactly the opposite situation arises in the 18 bonds of the central ring that are shared with the two types of six-membered rings on the periphery. In such bonds the small clockwise circulation $(0.265)$ due to the central ring is re-enforced by a con-

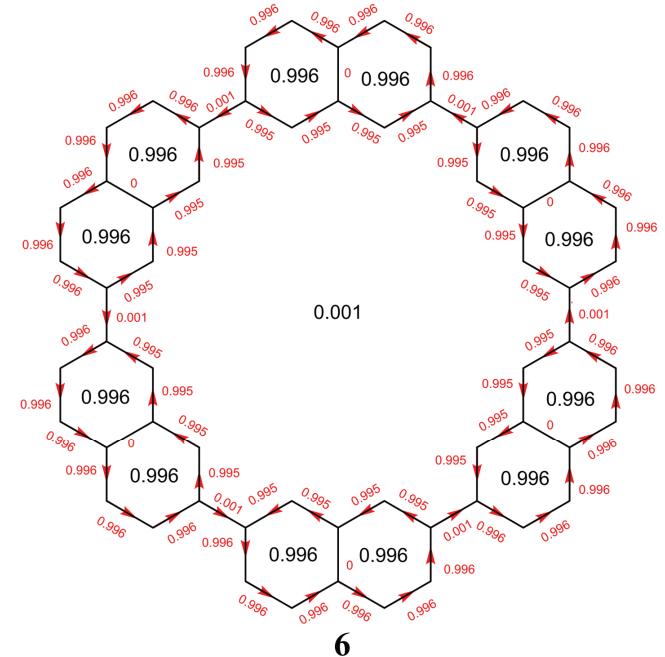

Figure 7. Ring-current and bond-current maps for structure 6 (named [6]cyclo-2,7-naphthylene by Nakanishi et al. ${ }^{53}$ ). For conventions on the representation of ring currents (black) and bond currents - red, in the on-line version of the journal please see the caption to Figure 3.

tribution (likewise in the clockwise - paramagnetic direction, from the point of view of the central ring) of either 0.239 or 0.056 , from the two sets of symmetrically distinct six-membered rings on the periphery. Consequently, the 18 bonds shared between the central ring and the two types of six-membered rings all carry currents which, from the point of view of the central ring, are in the clockwise (paramagnetic) direction. Accordingly, the sense of current flow in the central ring, like that around the perimeter, can be said to be 'mixed' (Table 1). (For quantitative details, please see the bond-current map for structure $\mathbf{5}$ depicted in Figure 6.) The nature of the current behaviour in the central ring of $\mathbf{5}$ is thus also inconsistent with the provisions of the AWA 'rule'.

Structure 6 (Figure 7). [6]Cyclo-2,7-naphthylene ${ }^{53}$ (6) may be thought of as being a naphthalenic analogy of hexa- $m$-phenylene (structure (4) of Ref. 1), which was included in Aihara's lis $\mathrm{t}^{32}$ of super-ring ${ }^{1}$ molecules (all depicted in Figure 2 of Ref. 1), with naphthalenic units in 6 (Figure 7) replacing the benzenoid ones in structure 4 of Ref. 1. It is, however, fundamentally different from the other six structures that have so far been considered here. Six of its bonds, which are all formally single and effectively carry zero ring-current, may be considered to form a part of both the inner ring and the outer ring. As can be seen from Figure 7, [6]cyclo-2,7-naphthylene (6) essentially consists (from a ring-current point of view) of six, completely isolated naphthalenic units, formed of two fused benzenoid rings (each bearing a ring current of intensity within $1 \%$ of the benzene value), and a $30-$ membered inner-ring supporting (to three decimal-places) zero ring-current, according to a Single-Precision calcu 
lation that was carried out, and a negligible 0.001, according to the calculation carried out in Double Precision, the results of which are shown in Figure 7. (Please see Ref. 1 for a discussion on the relative merits and drawbacks of Single-Precision and Double-Precision calculation in this context.) Like hexa-m-phenylene (structure 4 of Ref. 1), [6]cyclo-2,7-naphthylene (6) is one of those structures for which a continuous spanningtree cannot be drawn; ${ }^{1}$ accordingly, both the SinglePrecision and the Double-Precision calculations being discussed here were necessarily based on a branched spanning-tree. ${ }^{1}$ ) The same phenomenon was observed with [6]cyclo-2,7-naphthylene (6) as was noted when studying hexa- $m$-phenylene (structure (4) of Ref. 1): namely that, when the calculation was performed in Single Precision (in which intermediate quantities were handled to five decimal-places), a ring-current intensity of zero (to three decimal-places) was obtained for the 30-membered central-ring, but when a Double Precision calculation was undertaken (in which intermediate quantities were handled to ten decimal-places) this value was very slightly non-zero $(0.001$, to three places of decimals - precisely the same behaviour, incidentally, as that observed for the analogous central-ring of structure 4 of Ref. 1, when calculations were effected in Single or in Double Precision).

The outer ring ([4m+2], $m=10$ ) of $\mathbf{6}$ is seen (Figure 7) to exhibit diamagnetic bond-currents (of magnitude $c a$. 1.0) in 36 of its bonds, and virtually no current at all in the remaining six of its bonds. The central ring is likewise [4n+2]-membered (with, here, $n=7$ ). That ring's essentially zero ring-current does little to oppose the current of $c a$. 1.0 extant in the bonds that it shares with the six-membered rings. Hence, this central, 30membered ring bears clockwise (i.e., paramagnetic) currents in 24 of its bonds, and what may be considered to be virtually zero current in its remaining six bonds.

Thus, as was the case with hexa- $m$-phenylene (structure (4) of Ref. 1), even on the somewhat limited interpretation of [6]cyclo-2,7-naphthylene (6) as a 'superring' molecule ${ }^{1}$ that has been adopted here, this latter structure should also be considered to violate the AWA model.

Structure 7 (of Figure 2) The inner ring and the outer perimeter of cyclic [3]naphthylene ${ }^{52}(7)$ are both oddmembered and, as a consequence, this hydrocarbon is non-alternant, the only hydrocarbon considered in this study to which the provisions of the CoulsonRushbrooke Theorem ${ }^{5,56}$ do not apply. The structure consists of a [9]-membered central-ring within a [21]membered peripheral-ring. - i.e., it is a $[4 n+1]$-withina-[4m+1] system - as depicted in structure 7 of Figure 2. Like $\mathbf{5}$, this structure was also recently discussed by Balaban and Vollhardt. ${ }^{52}$
Before turning attention to 7, we first consider two other super-ring structures that have odd-membered perimeters and central-rings and whose compliance, or otherwise, with the AWA rule has already been investigated: ${ }^{14,20,22,57}$ these are corannulene (10) and [7]circulene (11), the carbon-atom skeletons of which are shown in Figure 8. Their quantitative topological ringcurrent and bond-current maps are both illustrated (labelled $\mathbf{2}$ and $\mathbf{3}$, respectively) in Figure 1 of Ref. 14. (The topological ring-current map for $\mathbf{1 0}$ was initially reported by one us many years ago $;^{57}$ it was of contemporary interest because the carbon-atom connectivity of corannulene is to be found on the surface ${ }^{58}$ of the then recently discovered buckminsterfullerene.) Current-density maps
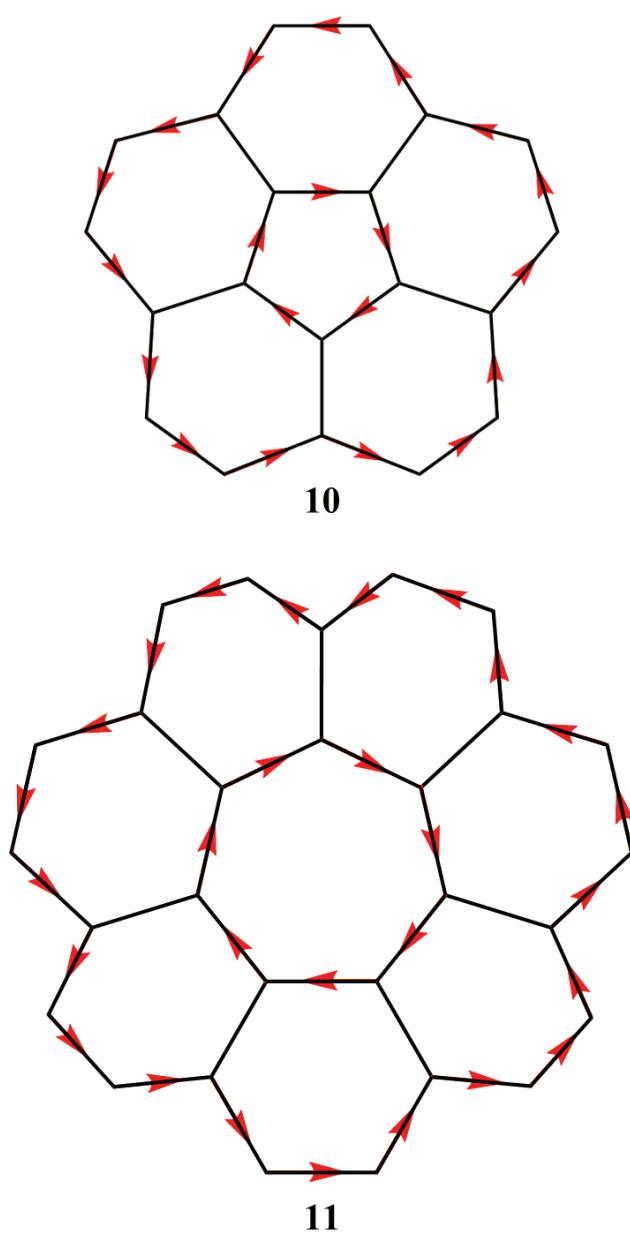

Figure 8. The carbon-atom connectivities of, and directions of bond-current flow in, (top) corannulene (10) (also known ${ }^{22}$ as [5]-circulene), which is a [5]-membered ring within a [15]-membered ring, and (bottom) [7]-circulene (11), which consists of a [7]-membered ring within a [21]-membered ring. The arrows indicate the direction of the topological bondcurrents in $\mathbf{1 0}$ and 11, which were quantitatively reported in Refs. 57 and 14. 
devised for $\mathbf{1 0}$ and $\mathbf{1 1}$ by application of the ipso-centric $a b$-initio formalism ${ }^{20-23,36}$ have been presented in Refs. 20 and 22.) For the purposes of the present discussion, topological bond-currents for $\mathbf{1 0}$ and $\mathbf{1 1}$ are qualitatively illustrated in Figure 8.

Barth and Lawton first synthesised dibenzo[ghi, $m n o]$ fluoranthene, which they named 'corannulene' and which is also known ${ }^{22}$ as [5]-circulene (structure $\mathbf{1 0}$ of Figure 8), nearly fifty years ago. ${ }^{18}$ As is seen from Figure 8 , the hydrocarbon is a super-ring system with a [5]membered central ring and a [15]-membered perimeter. Barth and Lawton rationalised ${ }^{18,19}$ the apparent stability of this species by explicitly devising the AWA model for the express purpose of discussing corannulene and postulating that, by transfer of one electron from the outer region to the central ring, ${ }^{18,57}$ corannulene could be modelled ${ }^{20}$ as a $6-\pi$-electron cyclopentadienyl anion within a $14-\pi$-electron annulenyl cation. As Steiner et $a l^{20}$ have pointed out, this assumption would require what they call 'con-rotatory rim and hub currents ${ }^{20}-$ that is, currents circulating in the same sense (and, here, it has to be the anti-clockwise sense - diamagnetic) around both the inner ring (which, by analogy with a wheel, they call the ${ }^{\prime h u b}{ }^{, 20}$ ) and the outer perimeter (what they call the 'rim" ${ }^{20}$ ). In practice, ipso-centric $a b$ initio ring-current calculations, ${ }^{20,36}$ as well as those ${ }^{14,57}$ based on the topological HLPM formalism, ${ }^{1-8}$ contradict this requirement: there is agreement ${ }^{14,20,57}$ between these two approaches (ab-initio 'ipso-centric ${ }^{20}$ and HLPM 'topological ${ }^{1-8,20,57,58}$ ) of considerably different sophistication that there is a parametric circulation in the central ring and a diamagnetic one around the perimeter. Other theoretical studies ${ }^{59,60}$ have likewise since cast doubt on the validity of Barth and Lawton's original hypothesis, ${ }^{18,19}$ invoked in order to account for the stability of corannulene (10).

Another system with an odd-membered inner-ring and perimeter that has been studied by both ipso-centric $a^{a}$-initio $^{22}$ and HLPM topological ${ }^{14}$ methods is [7]circulene (structure 11 of Figure 8). This has a [7]membered central-ring surrounded by a [21]-membered outer-ring. If there were electron transfer from the inner ring to the outer region, a $6-\pi$-electron system would be within a $22-\pi$-electron system. If the AWA rule applied, 'con-rotatory rim and hub currents ${ }^{20}$ would again be expected and - as these electron systems would both be of the form $[4 p+2]$ - the required 'con-rotatory' currents would need to be in the anti-clockwise (diamagnetic) sense in each circuit. In practice, however, when currents are calculated by both ipso-centric $a b$ initio $^{36}$ and topological ${ }^{1-4} \mathrm{HLPM}^{5-8}$ approaches, a paramagnetic circulation is found in the central ring and a diamagnetic one in the outer perimeter (as was the case with corannulene), thereby again apparently confounding the proposition about 'electron migration' suggested

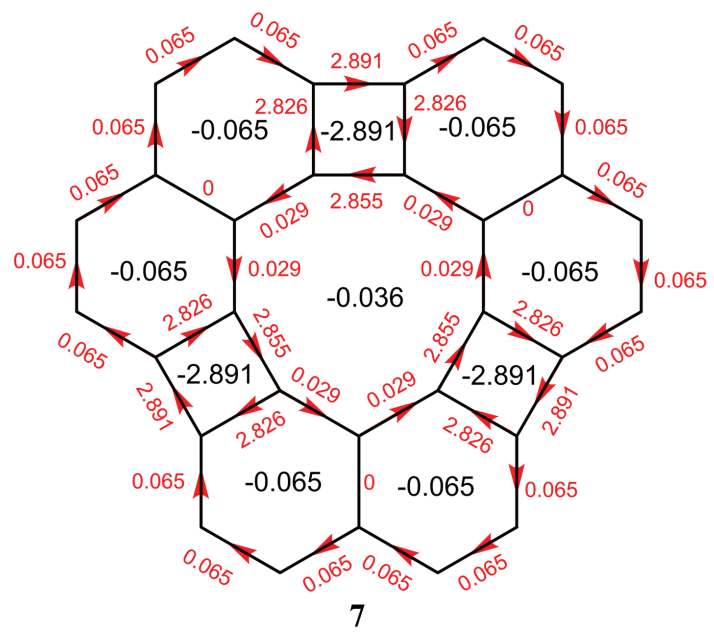

Figure 9. Ring-current and bond-current maps for structure 7 (called cyclic [3]naphthylene by Balaban and Vollhardt ${ }^{52}$ ). For conventions on the representation of ring currents (black) and bond currents - red, in the on-line edition of the journal - please see the caption to Figure 3.

above. The directions of these bond-current flows in $\mathbf{1 0}$ and $\mathbf{1 1}$ are indicated in Figure 8.

We now examine structure 7 of Figure 2, cyclic [3]naphthylene, ${ }^{52}$ a $9-\pi$-electron system within a $21-\pi$ electron system. Postulated electron-transfer from the inner to the outer region would yield an 8 - $\pi$-electron arrangement within a $22-\pi$-electron one. This time, only one circuit of the ' $[4 p+2]$ ' type would be created by this imagined process. It would result in the supposition of a '[4n]-within-[4m+2]' pattern, requiring 'counterrotatory currents', ${ }^{20-22}$ with a parametric circulation in the centre and a diamagnetic one around the perimeter. A glance at the current map for structure 7 shown in Figure 9 reveals that the currents are indeed 'counter' (that is, they are in the opposite senses) but it is the central ring that supports a net anti-clockwise (diamagnetic) circulation - though one which, at times, disappears almost to zero - and the perimeter that bears a (likewise very variable) current in the clockwise (paramagnetic) sense.

In this case, though, there is another notional 'electron-transfer' that could create a single circuit of the '[ $4 p+2]$ ' type. This is if the supposed transfer were in the opposite direction to that just proposed - this time from the outer region to the central ring. This would give rise to the idea of a [10]- $\pi$-electron system within a [20]- $\pi$-electron one. This implies a '[4n+2]within-[4m]' pattern, again requiring 'counter-rotatory currents, ${ }^{, 20-22}$ but, this time, with a diamagnetic circulation in the centre and a paramagnetic one around the perimeter. This is in fact what is observed, in the bondcurrent map for cyclic [3]naphthylene (7) depicted in Figure 9 - albeit, once again, with very uneven currents around the several bonds of both the central ring 
and the outer perimeter. An argument could, therefore, be advanced that 7 conforms to the AWA rule. However, this (a) relies on the possibly unwarranted assumption about the 'electron migration' that has to be postulated, and (b) in any case the magnitudes of the currentflows in both the central ring and the outer perimeter are extremely intermittent, decreasing almost to zero in six of the nine bonds in the central ring and in 18 of the 21 bonds around the perimeter

\section{CONCLUSIONS}

It is evident from the above discussion, summarised in Table 1, that, effectively, none of the structures 1-7 in Figure 2, whose $\mathrm{HLPM}^{5-8} \pi$-electron-current maps are depicted in Figures 3, 5-7 and 9, is in accord with the AWA rule. There is therefore a growing body of evidence to support the view that compliance with the AWA model is the exception, rather than the rule ${ }^{1,13-17}$ and that the model is "...by no means universal' ${ }^{1}$, is '...certainly not generally valid...', ${ }^{23}$ is '...no longer tenable.... ${ }^{22}$ and is '...a misnomer'. ${ }^{15,16}$ This appears especially to be true of those super-ring structures in which the outer and inner rings are what have been described as 'coupled'. ${ }^{13-16,23,34}$ Whether a super-ring system is described as 'coupled' or 'decoupled' depends on the nature of the 'spokes' bonds ${ }^{34}$ in what Monaco et $a l .^{23}$ call the 'cartwheel' that these super-ring structures may be considered to represent ( $c f$. Figure 1). These bonds are also known as 'transverse' 30 bonds and they have also been called 'radial' bonds ${ }^{20}$ they connect the central ring (what Fowler et al. call the 'hub ${ }^{, 21-23}$ ) to the outer perimeter (the 'rim'), as in Figure 1. In decoupled conjugated hydrocarbons of this type, these transverse/spokes/radial bonds never appear as anything other than single bonds in any Kekulé structure that can be devised for the system as a whole; a consequence of this is that, in these decoupled super-ring conjugated species, there are only ever four $(=2 \times 2)$ distinct Kekulé structures - such as are illustrated for a typical species of this type in Figure 2 of Ref. 15. Structures of this kind are specific examples of a more-general category of conjugated systems that Monaco and Zanasi ${ }^{26}$ have classified as 'molecules with a factorisable Kekulé count' so that if, in the case of a general 'super-ring' molecule, $K$ be the total number of Kekule structures for the system as a whole, this may be expressed as $K=$ $K_{\text {hub }} \times K_{\text {rim }}$, where $K_{\text {hub }}$ and $K_{\text {rim }}$ are the number of Kekulé structures for the central ring and the outer perimeter, respectively. Furthermore, by an ingenious corollary to Kasteleyn's Theorem, ${ }^{61}$ Monaco and Zanasi ${ }^{26}$ were able to identify the circumstances under which this state of affairs would arise. Such transverse/spokes/radial bonds in decoupled super-ring species all have Pauling bond-orders ${ }^{62,63,15}$ of zero. ${ }^{34}$ It should be noted, there- fore, that the following five statements that could be made about a super-ring ${ }^{1,30-33}$ system are all equivalent:

(a) The system is decoupled. ${ }^{1}$

(b) Its 'spokes' bonds (see Figure 1) invariably appear only as single bonds in any Kekulé structure that may be devised for the system as a whole.

(c) The Pauling bond-orders ${ }^{15,34,62,63}$ of the 'spokes' bonds are all zero.

(d) The system has precisely four Kekulé structures.

(e) The system has a factorisable Kekulé count, ${ }^{26}$ $K$, with - for 'two-layered 'super-ring' systems such as are considered here and in Ref. 1 $-K=K_{\text {hub }} \times K_{\text {rim }}$; (in the case of such 'twolayered ${ }^{17,27}$ 'super-ring ${ }^{1,30-33}$ systems, this is always $(2 \times 2)=4)$.

Now, it can, however, also be shown directly, by actually succeeding in drawing Kekulé structures in which one or more 'spokes' bonds are depicted as 'double' — and thereby creating practical counterexamples — that none of the super-ring conjugated systems (1-7 and $\mathbf{1 0}$ and 11) considered in this study is decoupled. We further note that none of them conforms to the AWA 'rule'.

Finally, we present one more structure of some considerable interest in that it formally represents an exception to the trend observed in the coupled Structures $\mathbf{1}-\mathbf{7}$. This is phenylene-[5]-circulene (12), whose HLPM ring-current and bond-current map is depicted in Figure 10 and which was discussed by Balaban and Vollhardt in Ref. 52. This structure, like [10,5]coronene, consists of a [10]-ring inside a [20]-ring and its main interest lies in the fact that it is an alternant isomer of the much-discussed ${ }^{15,16,23,25,26,30}$ (and decoupled $\left.^{15,16,23}\right)[10,5]$-coronene which, both ab-initio ${ }^{23}$ and HLPM calculations ${ }^{15,16}$ agree, respects the AWA rule. Unlike [10,5]-coronene, however, phenylene-[5]-circulene (12) is coupled - and therein lies its intrinsic interest, in this context.

It will be seen from the ring-current and bondcurrent maps (Figure 10) that all the ring currents in the central ten-membered ring and in all the four- and sixmembered rings of $\mathbf{1 2}$ are in the paramagnetic (clockwise) direction. This guarantees a paramagnetic circulation around the outer perimeter, as is required by the AWA model. The central ten-membered ring bears a paramagnetic current of -0.568 . This is (only just) smaller in magnitude than both of the paramagnetic ring-currents in the peripheral four-membered and sixmembered rings, with which the central [10]-membered ring shares bonds. These outer rings thus hold sway when it comes to competition in the bonds that these rings share with the central ring. As a consequence, in the bonds shared by the four-membered peripheral-rings and the central ring, the central ring's paramagnetic ring-current (of size 0.568 ) is easily swamped by the 


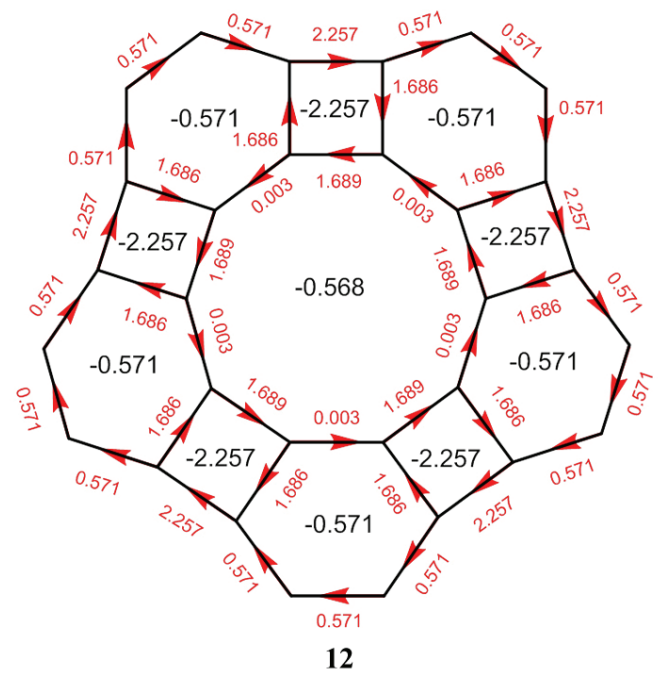

Figure 10. Ring-current and bond-current maps for structure 12 (called phenylene-[5]-circulene by Balaban and Vollhardt ${ }^{52}$ ), a (coupled) alternant isomer of [10,5]-coronene $e^{15,16,23,25,26}$ (which itself is decoupled). For conventions on the representation of ring currents (black) and bond currents - red, in the on-line edition of the journal - please see the caption to Figure 3.

four-membered rings' paramagnetic current of size 2.257 and so the direction of current flow in those bonds, so far as the $([4 n+2])$ central ring is concerned, is counter-clockwise (that is, in the diamagnetic direction). When it comes to the competition between the central ring (with its paramagnetic ring-current of magnitude 0.568 ) and the outer six-membered rings, however, the outer rings (with paramagnetic currents of size 0.571) only just prevail and so there is a very small current (of magnitude only $0.571-0.568=0.003$ ) in the diamagnetic direction (from the central ring's point of view) in those bonds of the central ring that are shared with six-membered rings on the periphery. The result is that - on what might almost be regarded as a 'technicality' — phenylene-[5]-circulene (12), like its decoupled non-alternant isomer [10,5]-coronene, does obey the AWA rule in that there is a net (though admittedly extremely intermittent) anti-clockwise circulation in the central ten-membered ring, and a clockwise one around the perimeter. This is the case despite the fact that phenylene-[5]-circulene (12) — like the structures 1-7 considered in the bulk of this paper - has an inner ring and an outer perimeter that may be regarded as coupled (because it is possible to draw Kekulé structures for $\mathbf{1 2}$ in which one or more 'spokes' bonds ${ }^{34}$ (see Figure 1) may formally be depicted as double bonds).

This is all in accord with the general conclusions drawn in Ref. 1 and elsewhere ${ }^{13-17,22,23}$ and this accumulating evidence seems to suggest that the Annulenewithin-an-Annulene model should either be used with considerable caution in very specific circumstances, or it should be abandoned altogether.

\section{APPENDIX}

It has been mentioned in the text that some of the structures considered here might possibly be non-planar either as a result of proton-proton steric-interactions and/or because of angle strain arising from too large a deviation from the idealised $s p_{2}$-hybridised $120^{\circ} \mathrm{C}-\mathrm{C}-\mathrm{C}$ angles.

In order to assess the importance of this point we have used the program ${ }^{64}$ Gaussian 09 (see also Refs. 65-67) to calculate the optimised geometry of structures 1 and 2 (Figure 2). The results are shown in Figure 11, which depicts the optimised geometries of those structures, as viewed from various aspects. It can be seen that $\mathbf{1}$ and $\mathbf{2}$ are not completely flat, but they appear not to be so distorted as entirely to prevent the notion of a ring current from being conceivable.

Now, the ring-current concept is strictly defined in the HLPM approach ${ }^{5-8}$ only for geometrically planar structures. ${ }^{68}$ Many years ago, however, in adapting the McWeeny method ${ }^{8}$ with the aim of making it approximately applicable to the non-planar helicene family, Haigh and one of the present authors ${ }^{69}$ stated that the assumptions required in order to do this were viable only if molecular overcrowding is such that '...the skeletal distortion about any bond is comparatively mild.' They further pointed out ${ }^{69}$ that, '...because of the way in which the strains from overcrowding are spread over

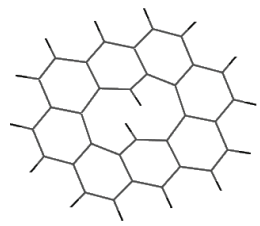

(a)

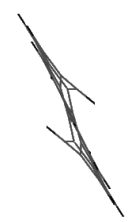

(b)

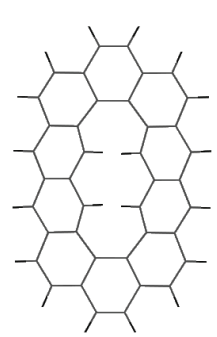

(c)

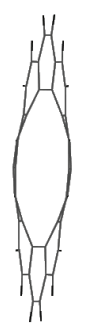

(d)

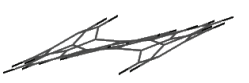

(e)
Figure 11. Views, from several aspects, of the geometryoptimised structure $\mathbf{1}$ of Figure 2 ((a) and (b)) and of structure 2 of Figure 2 ((c), (d) and (e)), as calculated by Gaussian 09 by use of the $6-311 \mathrm{G}$ basis set. ${ }^{64}$ 
many degrees of freedom, this condition does appear to be satisfied in the case of the helicenes, even though the overall non-planarity between well-separated parts of such molecules may in fact be very large.'

Figure 11 lends weight to the idea that similar considerations might apply in the case of structures $\mathbf{1}$ and 2. That said, however, it should be borne in mind that recent work in this area ${ }^{1-4}$ has treated the so-called topological ring-currents and bond-currents in conjugated systems - even ones that are manifestly non-planar ${ }^{2}$ - as a purely mathematical concept, based on the assumptions

(i) of a geometrically planar carbon-carbon framework and

(ii) of ring areas that are associated with regular polygons of unit side-length.

Accordingly, even though assumption (ii), above, has been relaxed in the case of the central rings of 1-4 and $\mathbf{6}$, the HLPM ring-currents ${ }^{1-4}$ calculated for any conjugated systems dealt with here that might be nonplanar are, in effect, those for a hypothetical structure having the same carbon-carbon connectivity as the actual molecule under consideration, but envisaged as if it were geometrically planar.

\section{REFERENCES}

1. T. K. Dickens and R. B. Mallion, Croat. Chem. Acta 86 (2013) 387-406.

2. R. B. Mallion, Croat. Chem. Acta 81 (2008) 227-246.

3. A. T. Balaban, T. K. Dickens, I. Gutman, and R. B. Mallion, Croat. Chem. Acta, 83 (2010) 209-215.

4. T. K. Dickens and R. B. Mallion, J. Phys. Chem. A 115 (2011) 351-356.

5. C. A. Coulson, B. O'Leary, and R. B. Mallion, Hückel Theory for Organic Chemists, Academic Press, London, 1978.

6. F. London, J. Physique Radium (7ème Série) 8 (1937) 397-409.

7. J. A. Pople, Mol. Phys. 1 (1958) 175-180.

8. R. McWeeny, Mol. Phys. 1 (1958) 311-321.

9. J. A. N. F. Gomes and R. B. Mallion, The Concept of Ring Currents, in: D. H. Rouvray (Ed.), Concepts in Chemistry, Research Studies Press Limited, Taunton, Somerset, England, United Kingdom, 1997, and John Wiley \& Sons, Inc., New York, 1997, Chapter 7, pp. 205-253.

10. J. A. N. F. Gomes and R. B. Mallion, Chem. Rev. 101 (2001) 1349-1383.

11. J. Aihara, R. Sekine, and T. Ishida, J. Phys. Chem. A 115 (2011) 9314-9321.

12. P. W. Fowler and W. Myrvold, J. Phys. Chem. A 115 (2011) 13191-13200.

13. T. K. Dickens and R. B. Mallion, J. Phys. Chem. A 115 (2011) 13877-13884.

14. T. K. Dickens and R. B. Mallion, Chem. Phys. Letters 517 (2011) 98-102.

15. T. K. Dickens and R. B. Mallion, Phys. Chem. Chem. Phys. 15 (2013) 8245-8253.

16. T. K. Dickens and R. B. Mallion, RSC Advances, 3 (2013) 15585-155588.

17. T. K. Dickens and R. B. Mallion, J. Phys. Chem. A 118 (2014) 933-939.

18. W. E. Barth and R. G. Lawton, J. Am. Chem. Soc. 88 (1966) $380-381$.
19. W. E. Barth and R. G. Lawton, J. Am. Chem. Soc. 93 (1971) 1730-1745.

20. E. Steiner, P. W. Fowler, and L. W. Jenneskens, Angew. Chem. 40 (2001) 362-366.

21. E. Steiner, P. W. Fowler, L. W. Jenneskens, and A. Acocella, Chem. Commun. (2001) pp. 659-660.

22. A. Acocella, R. W. A. Havenith, E. Steiner, P. W. Fowler, and L. W. Jenneskens, Chem. Phys. Letters 363 (2002) 64-72.

23. G. Monaco, R. Viglione, R. Zanasi, and P. W. Fowler, J. Phys. Chem. A 110 (2006) 7447-7452.

24. M. Lillington, P. W. Fowler, and M. Diudea, Polish J. Chem. 81 (2007) 653-662.

25. G. Monaco, P. W. Fowler, M. Lillington, and R. Zanasi, Angew. Chem. 46 (2007) 1889-1892.

26. G. Monaco and R. Zanasi, J. Chem. Phys. 131 (2009) 044126.

27. G. Monaco and R. Zanasi, J. Phys. Chem. A 116 (2012) 9020 9026.

28. G. Monaco, M. Memoli, and R. Zanasi, J. Phys. Org. Chem. 26 (2013) 109-114.

29. G. Monaco and R. Zanasi, Phys. Chem. Chem. Phys. 15 (2013) 17654-17657.

30. D. A. Bochvar, E. G. Gal'pern, and N. P. Gambaryan, Izv. Akad. Nauk SSSR, Ser. Khim., 3 (1970) 435-437. An English translation is available from: Consultants Bureau, A Division of Plenum Publishing Corporation, 227 West $17^{\text {th }}$ Street, New York, NY 10011, U.S.A., via the website: http://www.springerlink.com/content/15np5207n8q10240/

31. J. Aihara, J. Am. Chem. Soc. 114 (1992) 865-868.

32. J. Aihara, J. Phys. Chem. A 112 (2008) 4382-4385.

33. J. Aihara, M. Makino, T. Ishida, and J. R. Dias, J. Phys. Chem. A 117 (2013) 4688-4697.

34. A. T. Balaban, D. E. Bean, and P. W. Fowler, Acta Chim. Slov. 57 (2010) 507-512.

35. E. Steiner and P. W. Fowler, J. Phys. Chem. A 105 (2001) 9553 9562 .

36. E. Steiner, A. Soncini, and P. W. Fowler, J. Phys. Chem. A 110 (2006) 12882-12886.

37. P. W. Fowler and E. Steiner, Chem. Phys. Letters 364 (2002) 259-266.

38. S. Fias, P. W. Fowler, J. L. Delgado, U. Hahn, and P. Bultinck, Chem. Eur. J. 14 (2008) 3093-3099.

39. M Randić, Chem. Rev. 103 (2003) 3449-3605; (especially pp. 3539-3543).

40. M. Randić, Chem. Phys. Letters 500 (2010) 123-127.

41. M. Randić, D. Vukičević, and D. Plavšić, Ind. J. Chem. 88 (2011) 13-23.

42. M. Randić, Open Organic Chemistry Journal 5 (2011) 11-26.

43. M. Randić, D. Vukičević, A. T. Balaban, M. Vračko, and D. Plavšić, J. Comput. Chem. 33 (2012) 1111-1122.

44. M. Randić, M. Novič, M. Vračko, D. Vukičević, and D. Plavšić, Int. J. Quantum Chem. 112 (2012) 972-985.

45. M. Randić, D. Vukičević, M. Novič, and D. Plavšić, Int. J. Quantum Chem. 112 (2012) 2456-2462.

46. M. Randić, M. Novič, and D. Plavšić, J. Math. Chem. 50 (2012) 2755-2774.

47. M. Randič, Chem. Phys. Letters 38 (1976) 68-70.

48. J. A. N. F. Gomes, Some Magnetic Effects in Molecules, D. Phil. Thesis, University of Oxford, 1976, pp. 69-73.

49. J. A. N. F. Gomes and R. B. Mallion, Revista Portuguesa de Química 21 (1979) 82-89.

50. T. K. Dickens, J. A. N. F. Gomes, and R. B. Mallion, J. Chem. Theory Computation 7 (2011) 3661-3674.

51. I. Gutman and S. J. Cyvin, Introduction to the Theory of Benzenoid Hydrocarbons, Springer Verlag, Berlin (West) \& Heidelberg, 1989, pp. $127 \& 134$.

52. A. T. Balaban and K. P. C. Vollhardt, Open Organic Chemistry Journal 5 (2011) 117-126. 
53. W. Nakanishi, J. Y. Xue, T. Yoshioka, and H. Isobe, Acta Cryst. E67 (2011) o1752-o1763.

54. E. Clar, Nature 161 (1948) 239-239.

55. R. D. Broene and F. Diederich, Tetrahedron Letters 1991, pp. 5227-5230.

56. R. B. Mallion and D. H. Rouvray, J. Math. Chem. 5 (1990) 1-21; see also 'Postscript' 8 (1991) 399-400.

57. R. B. Mallion, Ring currents in Corannulene, a prototype patternmolecule for Buckminsterfullerene, in: A Graovac (Ed.), Math/Chem/Comp 1988. Proceedings of an International Course and Conference on the Interfaces between Mathematics and Computer Science, Dubrovnik, Yugoslavia, $20^{\text {th }}-25^{\text {th }}$ June, 1988 , Studies in Physical and Theoretical Chemistry, Elsevier Science Publishers B. V., Amsterdam, 1989, Vol. 63, pp. 505-510.

58. R. B. Mallion, Nature 325 (1987) 760-761.

59. G. Monaco, L. T. Scott, and R. Zanasi J. Phys. Chem. A 112 (2008) 8136-8147.

60. A. Sygula, J. Mol. Struct. THEOHEM 333 (1995) 215-226.

61. P. W. Kasteleyn, J. Math. Phys. 4 (1963) 287-293.

62. L. Pauling, L. O. Brockway, and J. Y. Beach, J. Am. Chem. Soc. 57 (1935) 2705-2709.

63. I. Gutman and S. J. Cyvin, Introduction to the Theory of Benzenoid Hydrocarbons, Springer Verlag, Berlin (West) \& Heidelberg, 1989, p 73.

64. Gaussian 09, Revision D.01, M. J. Frisch, G. W. Trucks, H. B.
Schlegel, G. E. Scuseria, M. A. Robb, J. R. Cheeseman, G. Scalmani, V. Barone, B. Mennucci, G. A. Petersson, H. Nakatsuji, M. Caricato, X. Li, H. P. Hratchian, A. F. Izmaylov, J. Bloino, G. Zheng, J. L. Sonnenberg, M. Hada, M. Ehara, K. Toyota, R. Fukuda, J. Hasegawa, M. Ishida, T. Nakajima, Y. Honda, O. Kitao, H. Nakai, T. Vreven, J. A. Montgomery, Jr., J. E. Peralta, F. Ogliaro, M. Bearpark, J. J. Heyd, E. Brothers, K. N. Kudin, V. N. Staroverov, R. Kobayashi, J. Normand, K. Raghavachari, A. Rendell, J. C. Burant, S. S. Iyengar, J. Tomasi, M. Cossi, N. Rega, J. M. Millam, M. Klene, J. E. Knox, J. B. Cross, V. Bakken, C. Adamo, J. Jaramillo, R. Gomperts, R. E. Stratmann, O. Yazyev, A. J. Austin, R. Cammi, C. Pomelli, J. W. Ochterski, R. L. Martin, K. Morokuma, V. G. Zakrzewski, G. A. Voth, P. Salvador, J. J. Dannenberg, S. Dapprich, A. D. Daniels, Ö. Farkas, J. B. Foresman, J. V. Ortiz, J. Cioslowski, and D. J. Fox, Gaussian, Inc., Wallingford, Connecticut, U.S.A., 2009.

65. S. F. Boys, Proc. Royal Soc. (London) Series A 200 (1950) 542 554.

66. S. F. Boys and N. C. Handy, Proc. Royal Soc. (London) Series A 310 (1969) 63-78.

67. W. J. Hehre, R. F. Stewart, and J. A. Pople, J. Chem. Phys. 51 (1969) 2657-2664.

68. C. W. Haigh, R. B. Mallion, and E. A. G Armour, Mol. Phys. 18 (1970) 751-766.

69. C. W. Haigh and R. B. Mallion, Mol. Phys. 22 (1971) 955-970. 